\title{
Active Control of Viscous Fingering Instabilities in Porous Media Flows
}

\author{
Jalel Azaiez \\ The University of Calgary \\ Canada
}

Instabilities at the interface between flowing solutions in a porous medium may take place as a result of viscosities and/or densities mismatch between the fluids involved in the flow displacement. These instabilities typically evolve in the form of fingers that extend in the flow and are referred to as viscous fingering or Saffman-Taylor instability in case of viscosities mismatch, and as density fingering or Rayleigh-Taylor instability in the case of densities mismatch. Such instabilities are encountered in a wide spectrum of industrial applications that include soil remediation, enhanced oil recovery and Carbon dioxide sequestration; and the underlying dynamics are also observed in a range of physical systems such as filtration combustion and the growth of bacterial colonies. Depending on the application, it is critical to be able to control the instabilities to either attenuate or enhance them. Different approaches that involve changes to the physical properties of the fluids (e.g. viscosity, density, surface tension) and/or the medium (e.g. permeability, wettability) have been so far explored as a controlling tool. In this talk, the use of the flow itself as a means to control the instability is examined. The advantage of this approach is that it does not require any alterations of the fluid/medium properties that can be either costly or simply unrealistic. Both linear stability analysis and numerical simulations are used to carry the study. A variety of flow displacements schemes for different flow geometries involving homogeneous porous media will be examined and the effects of different characteristics of the flow schemes on the instability are analyzed. These effects are analyzed both qualitatively and quantitatively to establish a direct link between the nature of the displacement scheme and the evolution of the viscous fingering instabilities. New interesting phenomena will be discussed and the potential for using the flow parameters and the instability structures as an indirect measure of the fluids physical properties will be proposed. 